\title{
Perfectly dissolved Boron nitride nanotubes due to polymer wrapping
}

Chunyi Zhi, ${ }^{\star}{ }^{\dagger}$ Yoshio Bando, ${ }^{\dagger \ddagger}$ Chengchun Tang, ${ }^{\dagger \ddagger}$ Rongguo Xie, ${ }^{\ddagger}$ Takashi Sekiguchi, ${ }^{\ddagger}$ and Dmitri Golberg ${ }^{\dagger \ddagger}$

\author{
${ }^{\dagger}$ Advanced Materials Laboratory, National Institute for Materials Science (NIMS), Namiki 1-1, \\ Tsukuba, Ibaraki 305-0044, Japan. \\ ${ }^{*}$ Nanomaterials Laboratory, National Institute for Materials Science. Namiki1-1, Tsukuba, \\ Ibaraki 305-004, Japan
}

RECEIVED DATE (automatically inserted by publisher); zhi.chunyi@nims.go.jp

\section{Support Information}

Figure S1. 1g of highly pure BNNTs. The color of BNNTs is pure white. 
Figure S2. SEM image of BNNTs. The purity of a sample can be more than 90 vol\%.

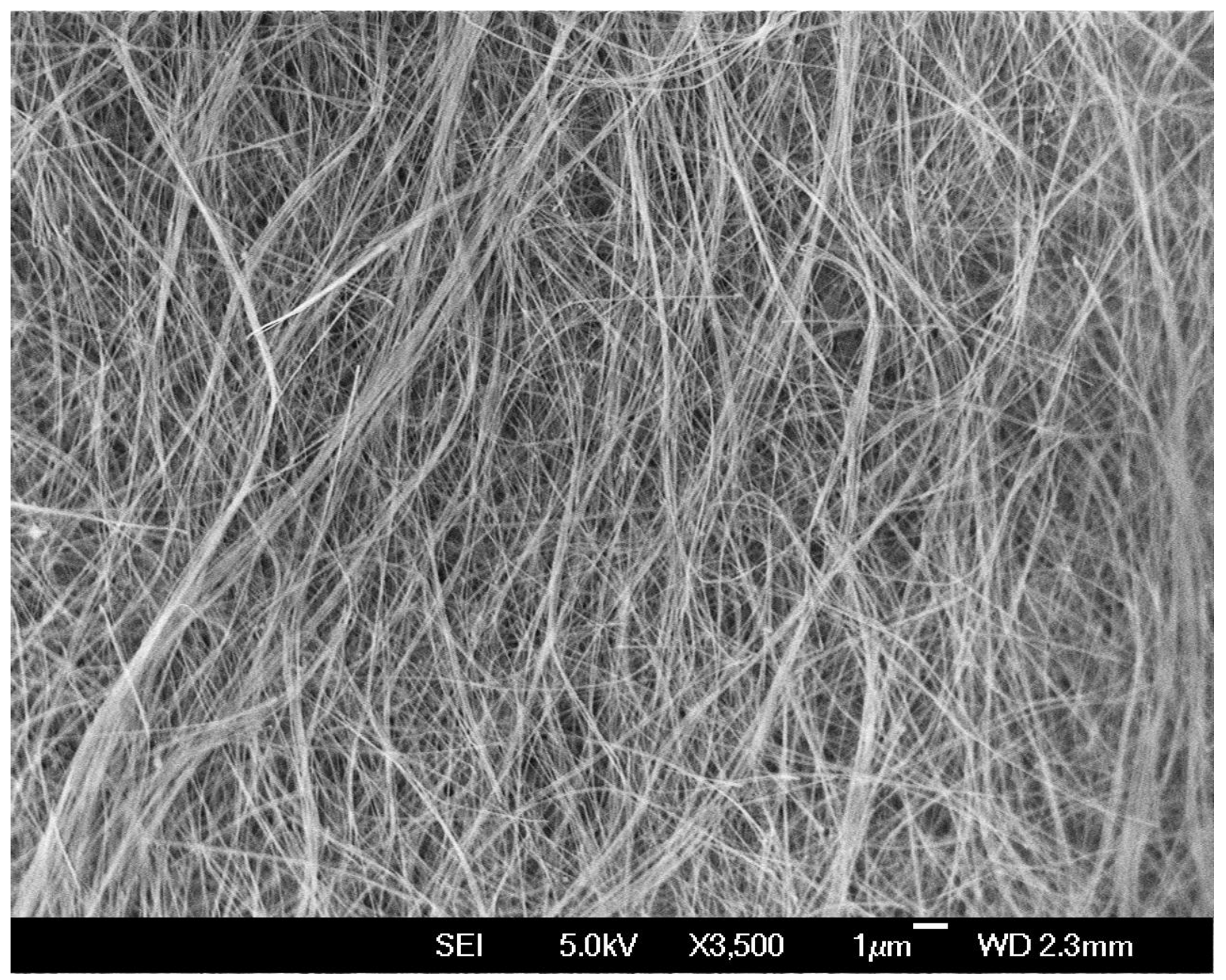


Figure S3. X-ray diffraction spectrum of BNNTs, all peaks can be indexed to $h$-BN phase.

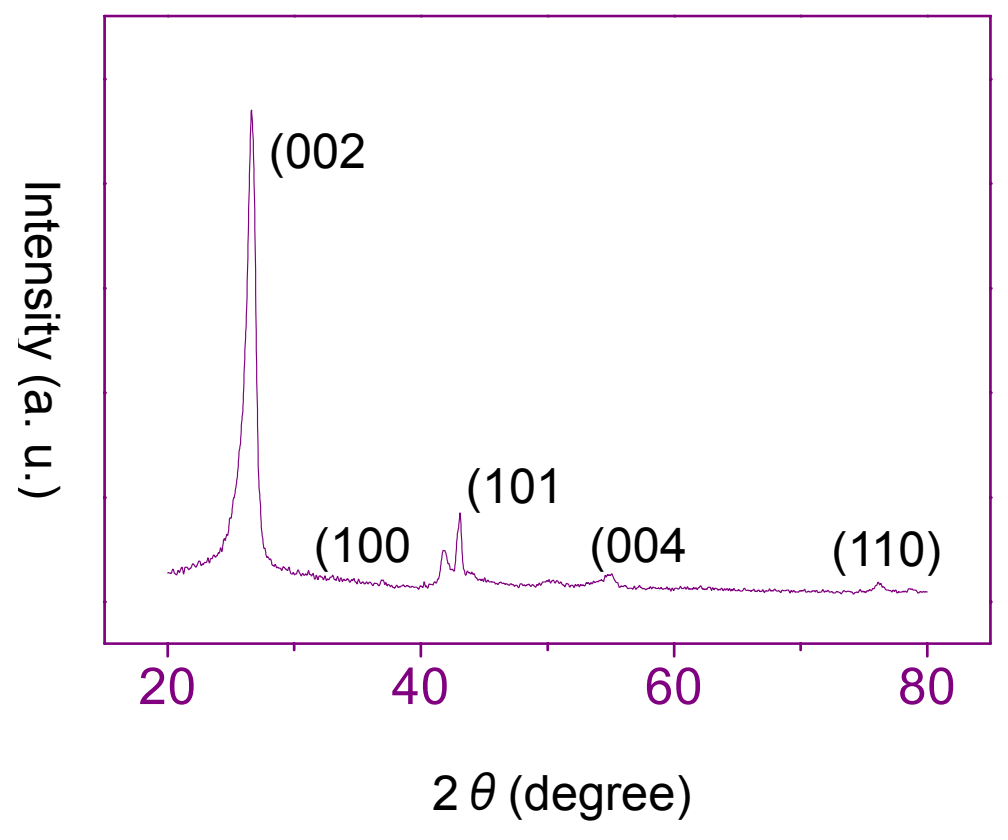


Figure S4. Thermogravimetric analysis of BNNT and CNT.

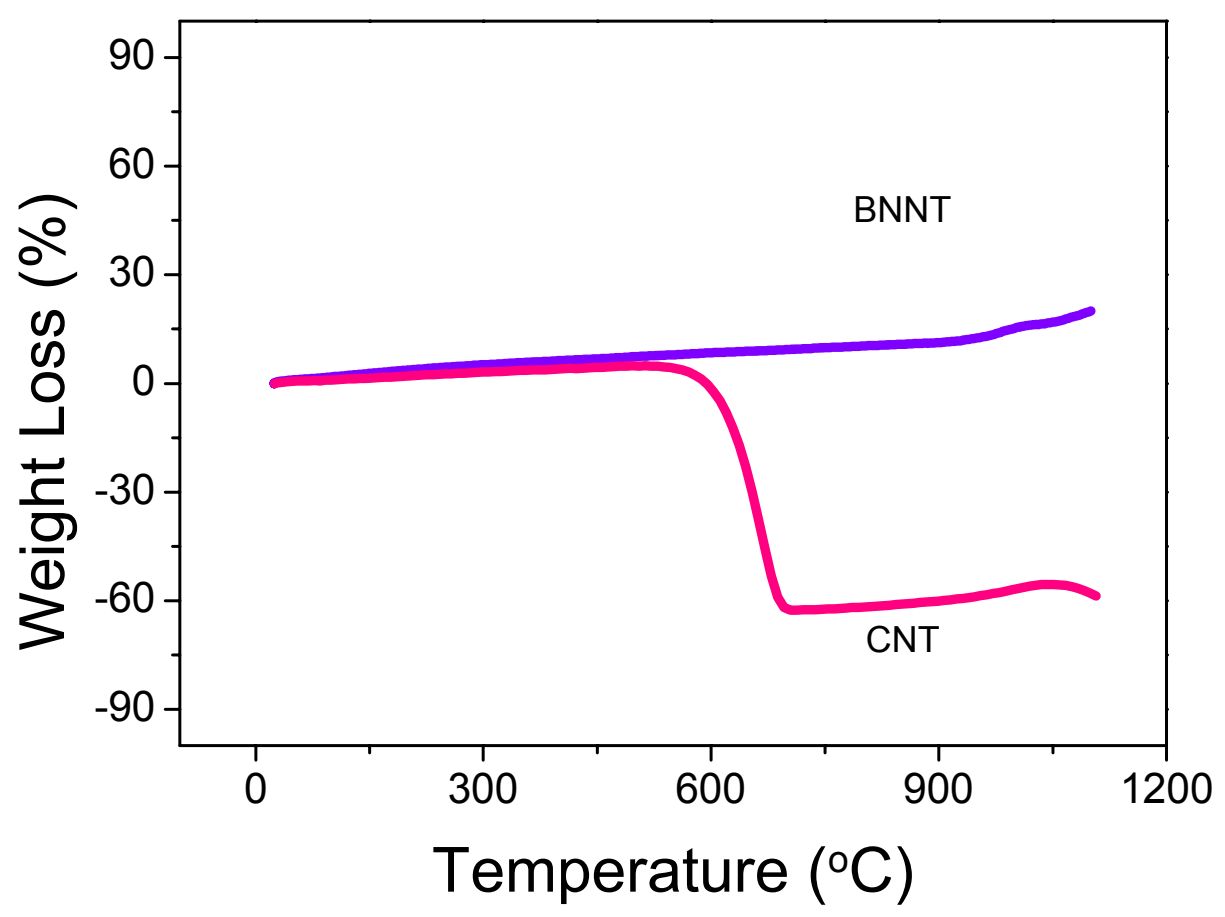


Figure S5 CL spectrum of BNNTs at 15K. A peak at around 5.5 $\mathrm{eV}$ is clearly visible.

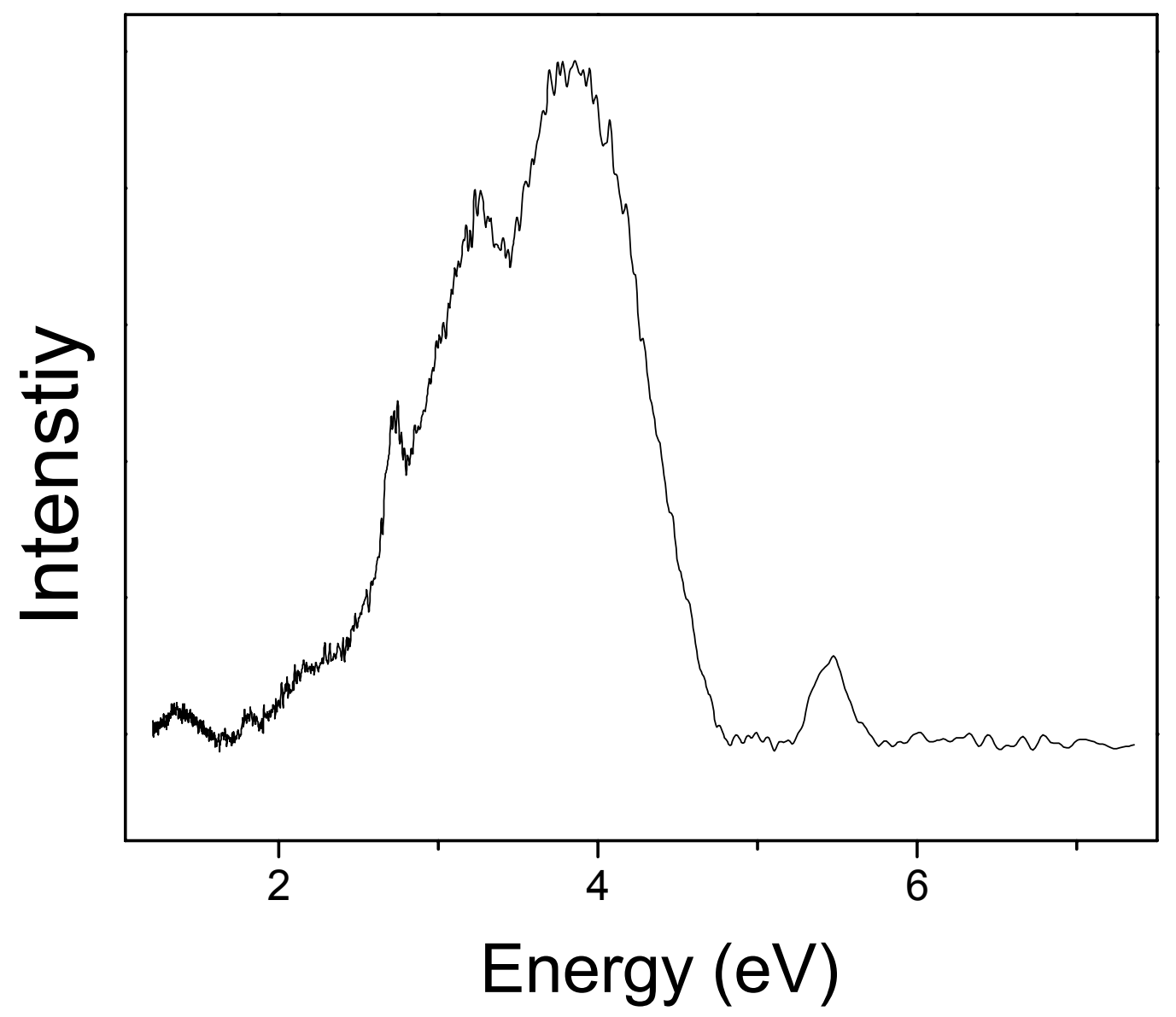


Figure S6. SEM image of a BNNT film. The sample holder was tilted to $60^{\circ}$. This image indicates that the thickness of the film is around $2 \sim 5 \mu \mathrm{m}$.

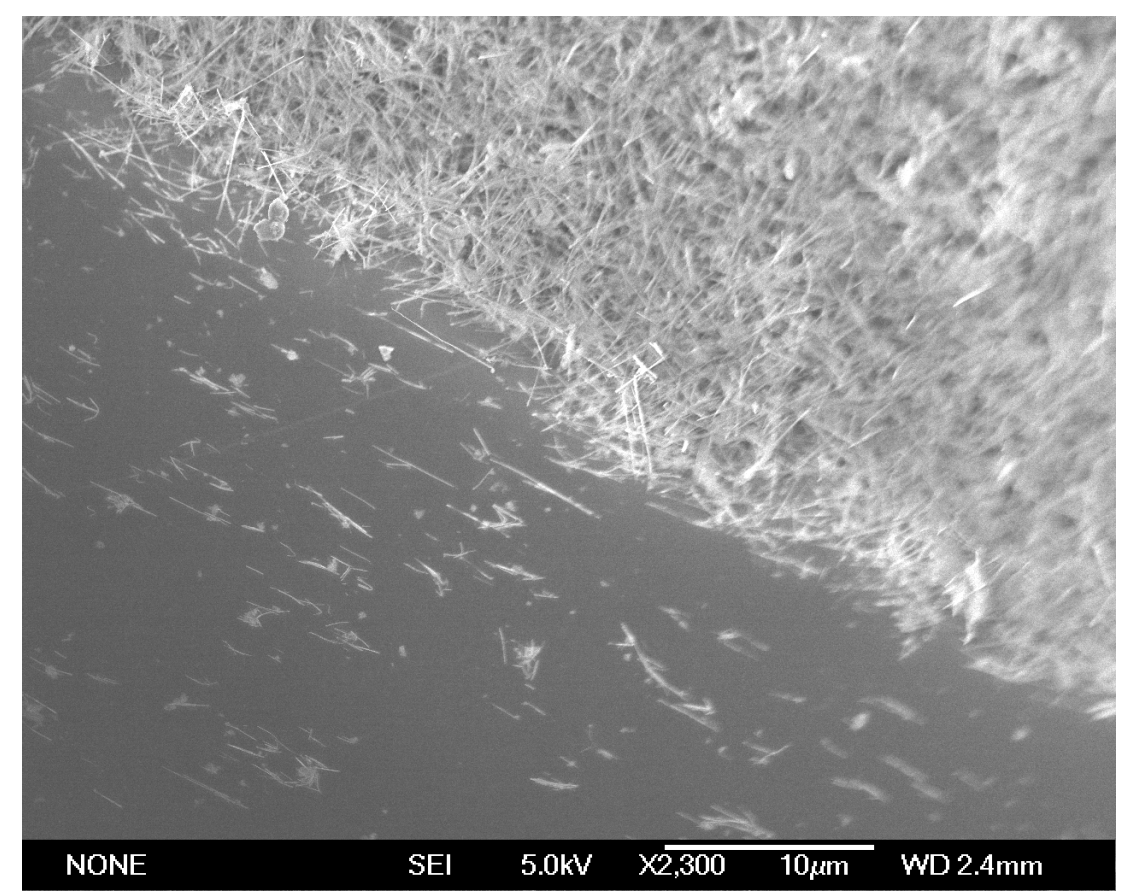

